ARQGA / 970

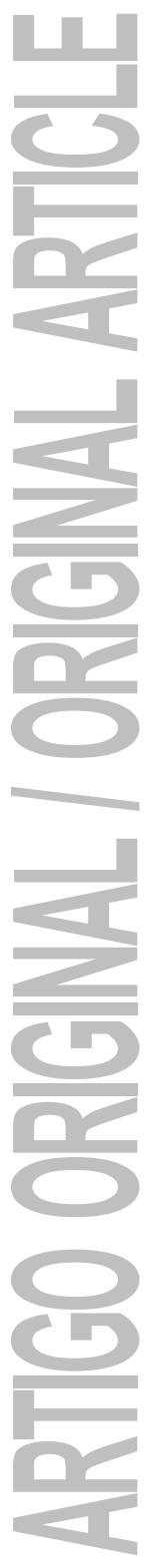

\title{
IMPORTÂNCIA DA ALBUMINA SÉRICA NA AVALIAÇÃO NUTRICIONAL E DE ATIVIDADE INFLAMATÓRIA EM PACIENTES COM DOENÇA DE CROHN ${ }^{+}$
}

\author{
Virgínia Lúcia Ribeiro CABRAL, Luciana de CARVALHO e \\ Sender Jankiel MISZPUTEN
}

\begin{abstract}
RESUMO - Racional - A albumina sérica é freqüentemente definida como um indicador de estado nutricional. No entanto, hipoalbuminemia pode refletir reação de fase aguda mediada por citocinas durante um evento inflamatório. Tanto desnutrição, como hipoalbuminemia são achados relevantes na doença de Crohn. Objetivo - Estudar a relação da albumina sérica com atividade inflamatória e com estado nutricional em pacientes com doença de Crohn. Casuística/Método Trinta e seis pacientes avaliados quanto ao estado nutricional por parâmetros antropométricos e a atividade inflamatória da doença intestinal definida por índice clínico e velociodade de hemossedimentação. Resultados - Não se encontrou associação entre desnutrição e hipoalbuminemia. A utilização dessa proteína como parâmetro bioquímico na avaliação nutricional foi dispensável para se diagnosticar desnutrição. Houve significativa relação entre atividade inflamatória e hipoalbuminemia, demonstrando $100 \%$ de sensibilidade na identificação de inflamação ativa. Conclusão - Estes resultados sugerem a dosagem de albumina sérica como marcador sensivel de doença de Crohn ativa e a necessidade de um parâmetro laboratorial mais adequado para avaliação nutricional rotineira nos portadores desta doença inflamatória intestinal.
\end{abstract}

DESCRITORES - Doença de Crohn. Albumina. Desnutrição. Inflamação. Citocinas.

\section{INTRODUÇÃO}

A doença de Crohn (DC) é considerada doença inflamatória intestinal (DII) sem etiopatogenia definida, caracterizando-se por processo inflamatório crônico com períodos de agudização, que pode comprometer todo o trato gastrointestinal. Aceita-se que, em indivíduos geneticamente predispostos, antígenos derivados da microflora e da dieta induziriam a uma resposta do sistema imune, ocasionando inflamação ${ }^{(9)}$.

Recentes estudos têm demonstrado a importância das citocinas na regulação da hematopoiese, cicatrização e inflamação ${ }^{(8,21)}$. Dentre elas, a interleucina-6 (IL-6), interleucina-1 (IL-1) e o fator de necrose tumoral $\alpha$ (TNF $\alpha$ ), são considerados os mediadores inflamatórios mais importantes ${ }^{(1)}$.

+ Departamento de Medicina Clínica, Disciplina de Gastroenterologia, Escola Paulista de Medicina - UNIFESP-EPM

Endereço para correspondência: Dra. Virgínia Lúcia R. Cabral - Rua Rafael Sampaio Vidal, 77 - 09550-170 - São Caetano do Sul, SP. 
Na vigência de inflamação, a IL-6 é responsável pelo aumento na síntese de proteínas plasmáticas, referidas como de fase aguda, e pela diminuição na produção de albumina e transtirretina ${ }^{(7,20)}$. O principal mecanismo regulador da síntese protéica ocorre por mudança na transcrição genética no hepatócito e não por menor aporte de aminoácidos ao fígado ${ }^{(2)}$.

Aumento na produção de citocinas pró-inflamatórias é achado relevante na doença de Crohn e na retocolite ulcerativa inespecífica. Níveis elevados de IL-1 $\beta$, TNF $\alpha$ e IL-6 foram encontrados na mucosa intestinal inflamada e em monócitos obtidos de sangue periférico dos portadores destas doenças ${ }^{(6,26,31)}$, sendo que na doença de Crohn, a IL-6 se manteve em maiores concentrações durante a atividade inflamatória ${ }^{(23)}$.

A hipoalbuminemia tem estado diretamente associada com estado nutricional, mais precisamente com desnutrição protéico-calórica (DPC), achado freqüente nos pacientes com doença de $\mathrm{Crohn}^{(13,19)}$. Da mesma forma, alterações da massa óssea e de crescimento em crianças portadoras desta doença inflamatória têm sido relacionadas com desnutrição, decorrente de alterações digestivas como, anorexia, dor abdominal, diarréia, má absorção e do uso de corticoesteróides ${ }^{(18)}$; no entanto, o aumento de citocinas pró-inflamatórias foi associado com mudanças no metabolismo ósseo, resultando em perda de massa óssea e déficit de crescimento ${ }^{(17)}$.

BOLOGA et al. ${ }^{(4)}$ identificaram associação entre níveis aumentados de IL-6, hipoalbuminemia e hipocolesterolemia em doentes renais crônicos que se mantinham em hemodiálise. Este achado é reforçado pela constatação de que a hipoalbuminemia encontrada em idosos hospitalizados por pneumonia se devia ao aumento de IL-6, com normalização de seus níveis com a cura do quadro infeccioso ${ }^{(15)}$.

\section{Objetivo}

Por se conhecer que a doença de Crohn cursa com períodos de agudização e que a albumina é considerada uma proteína de síntese negativa na fase aguda de inflamação, procurou-se definir, com mais clareza, o comportamento desta proteína neste grupo de pacientes.

\section{CASUÍSTICA E MÉTODOS}

Trinta e seis pacientes portadores de DC, dentre estes, um com colangite esclerosante primária (CEP) associada, tiveram suas doenças diagnosticadas por critérios clínicos, radiológicos, endoscópicos e morfológicos ${ }^{(22,25)}$ e foram subdivididos de acordo com sexo, idade, duração dos sintomas, estado nutricional, localização e atividade inflamatória da doença.

A presença de atividade inflamatória foi definida pelo índice clínico simples de HARVEY e BRADSHAW ${ }^{(14)}$. Considerou-se doença ativa, quando a soma dos parâmetros clínicos encontrava-se com escore maior ou igual a 5 .
Para definir localização da doença, utilizou-se exame radiológico do trânsito intestinal (delgado) e endoscópico com biopsia para trato digestivo alto e cólon.

\section{Avaliação Nutricional}

Os pacientes foram regularmente acompanhados por avaliação nutricional através de parâmetros antropométricos: altura, peso, peso ideal/peso atual (PI/PA), prega cutânea do tríceps (PCT), circunferência do braço (CB) e circunferência muscular do braço (CMB). Todos estes parâmetros foram adequados de acordo com o proposto por FRISANCHO $^{(10)}$, considerando-se o percentil 50 como valor ideal.

Para avaliação bioquímica quanto ao estado nutricional utilizouse a albumina sérica.

Definiu-se o escore de DPC pela soma dos valores obtidos destes parâmetros dividido pelo número de parâmetros estudados ${ }^{(24)}$.

Escore de DPC $\sum \%$ adequacão PCT, CB, CMB, (PI/PA) e albumina 5

$\mathrm{O}$ valor obtido permitiu classificar os pacientes de acordo com as recomendações de BLACKBURN et al..$^{(3)}$ em eutrofia e graus de DPC (leve, moderado e grave).

\begin{tabular}{ll}
\hline & DPC (\%) \\
\hline Eutrofia & $>100$ \\
Desnutrição & \\
Leve & $<100$ a 80 \\
Moderada & $<80$ a 60 \\
Grave & $<60$ \\
\hline
\end{tabular}

\section{Laboratório}

Coleta de sangue em veia periférica ocorreu no momento do interrogatório sobre sintomas relacionados à doença.

$\rightarrow$ Dosagem de albumina sérica: eletroforese de proteínas (acetato de celulose)

Níveis $\leq 3,5 \mathrm{~g} / \mathrm{dL}$ foram considerados como alterados, definindose os níveis de depleção como: 3,5 a $2,8 \mathrm{~g} / \mathrm{dL} \rightarrow$ discreta

$$
\begin{aligned}
2,7 \text { a } 2,1 \mathrm{~g} / \mathrm{dL} & \rightarrow \text { moderada } \\
<2,1 \mathrm{~g} / \mathrm{dL} & \rightarrow \text { grave }
\end{aligned}
$$

$\rightarrow$ Velocidade de hemossedimentação (VHS): Westegren

Apenas os valores $\geq 30 \mathrm{~mm} / \mathrm{h}$ foram valorizados, afim de minimizar a influência do sexo e /ou idade na análise deste parâmetro ${ }^{(28)}$.

\section{Método estatístico}

Variáveis qualitativas foram analisadas pelo teste do Qui-quadrado. Optou-se pelo teste exato de Fisher quando as restrições de Cochran 
impediram a utilização do Qui-quadrado. Para variáveis quantitativas utilizou-se o teste de Mann-Whitney.

O teste " $t$ " emparelhado comparou os resultados obtidos pelas avaliações nutricionais com e sem albumina sérica, como parâmetro laboratorial.

Valores de $P$ menores que 0,05 foram considerados significantes.

\section{RESULTADOS}

A distribuição dos pacientes com doença de Crohn de acordo com os valores encontrados de albumina sérica estão apresentados na Tabela 1.

TABELA 1 - Características demográficas e clínicas dos pacientes com doença de Crohn segundo a dosagem de albumina sérica

\begin{tabular}{lcc}
\hline \multirow{2}{*}{ Características } & \multicolumn{2}{c}{ Albumina sérica (g/dL) } \\
\cline { 2 - 3 } & $\leq 3,5$ & $>3,5$ \\
\hline Sexo (F/M) & $16 / 7$ & $5 / 8$ \\
Idade (anos) $\left.)^{*}\right)$ & 39 & 32,4 \\
Duração dos sintomas(meses) ${ }^{(*)}$ & 120 & 70 \\
Localização da doença & & \\
Delgado & 12 & 6 \\
Delgado/cólon & 6 & 6 \\
Cólon/reto & 5 & 1 \\
Atividade inflamatória & & \\
Ativa & 13 & 0 \\
Inativa & 10 & 13 \\
Av. nutricional & & \\
Eutrófico & 5 & 10 \\
Desnutrido & 18 & 0 \\
Associação com CEP & 1 & \\
\hline
\end{tabular}

(*) Média

Ao se considerar a avaliação nutricional, excluindo-se o parâmetro bioquímico albumina sérica, encontraram-se 28 pacientes desnutridos, sendo 16 com desnutrição leve e 12 com desnutrição moderada, resultando numa prevalência de $77,8 \%$. Correlacionando-se os valores encontrados de albumina sérica com a avaliação nutricional destes pacientes, baseada apenas nos dados antropométricos, não foi possível detectar associação entre hipoalbuminemia e desnutrição, mesmo quando os graus de desnutrição foram analisados isoladamente $(P=$ 1), definindo-se sensibilidade de $64 \%$ para albumina sérica identificar desnutrição e especificidade de 37,5\%. O mesmo ocorreu ao se pesquisar a relação entre desnutrição, diagnosticada apenas por parâmetros antropométricos e atividade inflamatória.

Não houve diferença estatística quando foram comparados os resultados obtidos pela avaliação nutricional, utilizando ou não valores de albumina sérica como parâmetro bioquímico $(P=1)$.
Ao se estudar o comportamento da albumina sérica em relação à atividade inflamatória, localização da doença e duração dos sintomas, houve associação inversa entre atividade inflamatória (índice clínico) e os valores de albumina sérica $(P<0,001)$, com sensibilidade para albumina detectar inflamação ativa de $100 \%$ e especificidade de $56,5 \%$. Quando o VHS $\geq 30 \mathrm{~mm} / \mathrm{h}$ representou doença ativa, relação com hipoalbuminemia $(P=0,01)$ também foi identificada. No entanto, não se observou correlação com a localização da doença (delgado/cólon) e com duração dos sintomas $(P>0,05)$.

\section{DISCUSSÃO}

A albumina é a proteína mais abundante no sangue. Apesar da complexidade de fatores que interferem em seu nível sangüíneo como, aporte de aminoácidos para sua síntese, perda, degradação, meia-vida longa e distribuição entre intra e extra-vascular ${ }^{(27)}$, ela continua sendo importante parâmetro bioquímico para avaliação de estado nutricional e de índice prognóstico em várias doenças, principalmente as hepáticas por sua forma de detecção ser de baixo custo e de fácil acesso.

Verificou-se alta prevalência de hipoalbuminemia e desnutrição nos pacientes desta série com doença de Crohn como referido por outros autores $^{(12,29)}$, mas não ocorreu associação entre estas duas características, inclusive definindo hipoalbuminemia como mau indicador de desnutrição.

A presença de desnutrição também não se relacionou com doença de Crohn ativa, não sendo possível confrontar os graus de desnutrição com a agressividade da doença, em decorrência da heterogeneidade do seu comportamento clínico e, conseqüentemente, da terapêutica empregada.

Todos os pacientes com doença de Crohn ativa (índice clínico), apresentavam hipoalbuminemia, sugerindo que a meia-vida da albumina (cerca de 20 dias) não interferiu neste resultado, embora o conhecimento do intervalo de tempo, entre o início da atividade inflamatória e a dosagem de albumina não tenha sido corretamente definido. Este resultado é semelhante ao referido por HYAMS et al. ${ }^{(16)}$ que detectaram hipoalbuminemia em crianças com doença de Crohn ativa e esta se relacionou diretamente com níveis elevados de IL-6.

Dez pacientes definidos como tendo doença inativa (índice clínico), apresentavam hipoalbuminemia, na ausência de má absorção, perdas entérica e/ou urinária. Destes, nove eram desnutridos, sendo cinco com desnutrição leve. Contudo este achado não se associou com o grau de depleção da albumina sérica.

Níveis séricos diminuídos de albumina também não se relacionaram com duração dos sintomas, localização e extensão da inflamação, embora sua associação com a forma de apresentação da doença, isto é, estenoses, fístulas e lesões peri-anais não tenha sido estudada, já que a maioria destes pacientes apresentava forma mista de manifestação. 
Cabral VLR, Carvalho L de, Miszputen SJ. Importância da albumina sérica na avaliação nutricional e de atividade inflamatória em pacientes com doença de Crohn

Sabe-se que, métodos utilizados rotineiramente para avaliação de atividade inflamatória na doença de Crohn podem não ser tão sensíveis como a pesquisa de citocinas pró-inflamatórias presentes durante a inflamação aguda ${ }^{(5)}$, subestimando assim, aqueles casos em atividade inflamatória "subclínica." Esta hipótese pode ser reforçada pela referência de GOMES et al. ${ }^{(11)}$ que encontraram alterações endoscópicas compatíveis com inflamação aguda em pacientes portadores de DII, dita como inativa pelos índices clínicos.

Deve-se ressaltar que a avaliação nutricional realizada por antropometria e parâmetros bioquímicos, quais sejam, albumina sérica, transferrina, transtirretina e capacidade de combinação do ferro, deveria ser acompanhada da pesquisa de atividade inflamatória, visto que as citocinas envolvidas na inflamação, modificam o comportamento destes parâmetros laboratoriais e, inclusive, a análise de massa muscular poderia também sofrer influência da proteólise e mesmo da atrofia decorrentes da ação da IL-6 ${ }^{(30)}$, agravada pela utilização, muitas vezes, de corticoterapia crônica ${ }^{(32)}$.

Podemos concluir que outros estudos são necessários para comprovar a importância da albumina sérica como marcador sensível de atividade inflamatória em adultos portadores de DC, possíveis fatores que interferem em sua especificidade e sua relação temporal com o aparecimento dos sinais clínicos de inflamação aguda.

A avaliação nutricional por parâmetros antropométricos e bioquímicos rotineiros deve ser realizada durante todo o acompanhamento clínico dos pacientes com doença de Crohn para evitar que uma análise transversal destes parâmetros sofra influência da ação de citocinas presentes durante uma agudização da doença.

Cabral VLR, Carvalho L de, Miszputen SJ. Importance of serum albumin values in both nutritional and inflammatory activity assessment in patients with Crohn's disease. Arq Gastroenterol 2001;38(2):104-108.

ABSTRACT - Background - The serum albumin concentration has frequently been regarded as an indicator of nutritional status, although the hypoalbuminemia may reflect an acute phase protein response during inflammation mediated by cytokines. Both hypoalbuminemia and malnutrition are observed in Crohn's disease. Objectives - To correlate the serum albumin values to disease activity and also to nutritional status in patients with Crohn's disease. Patients/Methods - Thirty six patients were studied. Nutritional status was assessed by anthropometry measures and inflammatory activity determined by Harvey's simple clinical index and erythrocyte sedimentation rate. Results - No correlation was found between malnutrition and hypoalbuminemia. The serum albumin levels correlated inversely with the disease activity. Hypoalbuminemia was $100 \%$ sensitive for detection of disease activity. Conclusion - This study suggests that serum albumin concentration is a very sensitive marker of inflammatory activity and not good indicator of the nutritional status in Crohn's disease. It is necessary a suitable laboratorial parameter for routine nutrition assessment in patients with this inflammatory bowel disease.

HEADINGS - Crohn disease. Albumin. Malnutrition. Inflammation. Cytokines.

\section{REFERÊNCIAS BIBLIOGRÁFICAS}

1. Andus T, Geiger T, Hirano T, Kishimoto T, Heinrich PC. Action of recombinant human interleukin-6; interleukin- $1 \beta$ and tumor necrosis factor $\alpha$ on the mRNA induction of acute-phase proteins. Eur J Immunol 1988;18:739-46.

2. Birch HE, Schreiber G. Transcriptional regulation of plasma protein synthesis during inflammation. J Biol Chem 1986;261:8077-80.

3. Blackburn G L, Bistrion BR, Moini BS, Schlamn HT, Smith MF. Nutritional and metabolic assessment of the hospitalized patient. JPEN J Parent Enteral Nutr 1977;1:11-22

4. Bologa RM, Levine DM, Parker TS, Cheigh JS, Serur D, Stenzel KH, Rubin AL Interleukin-6 predicts hypoalbuminemia, hypocholesterolemia, and mortality in hemodialysis patients. Am J Kidney Dis 1998;32:107-14.

5. Braegger CP, MacDonald TT. Immune mechanisms in chronic inflammatory bowel disease. Ann Allergy 1994;72:135-41.

6. Brynskov J, Tvede N, Andersen CB, Vilien M. Increased concentrations of interleukin-1 beta, interleukin-2 and soluble interleukin-2 receptors in endoscopical mucosal biopsy specimens with active inflammatory bowel disease. Gut 1992;33:55-8

7. Castell JV, Gómez-Lechón MJ, David M, Fabia R, Trullenque R, Heinrich PC Acute-phase response of human hepatocytes: regulation of acute-phase protein synthesis by interleukin-6. Hepatology 1990;12:1179-86.

8. Dinarello CA. Interleukin-1. Rev Infect Dis 1984;6:51-95.

9. Fiocchi C. Inflammatory bowel disease: etiology and pathogenesis. Gastroenterology 1998;115:182-205
10. Frisancho AR. New norms of upper limb fat and muscle areas for assessment of nutritional status. Am J Clin Nutr 1981:34:2540-5.

11. Gomes P, Boulay CD, Smith CL, Holdstock G. Relationship between disease activity indices and colonoscopic findings in patients with colonic inflammatory bowel disease. Gut 1986;27:92-5.

12. Harries AD, Heatley RV. Nutritional disturbances in Crohn's disease. Postgrad Med J 1983;59:690-7.

13. Harries AD, Jones LA, Danis V, Fifield R, Heatley RV, Newcombe RG, Rhodes J Controlled trial of supplemented oral nutrition in Crohn's disease. Lancet 1983;1:887-90.

14. Harvey RF, Bradshaw JM. A simple index of Crohn's disease activity. Lance 1980;8:514.

15. Hedlund JU, Hansson L-O, Örtquist AK. Hypoalbuminemia in hospitalized patients with community-acquired pneumonia. Arch Intern Med 1995; 155:143842.

16. Hyams JS, Fitzgerald JE, Treem WR, Wyzga N, Kreutzer DL. Relationship of functional and antigenic interleukin 6 to disease activity in inflammatory bowel disease. Gastroenterology 1993;104:1285-92.

17. Hyams JS, Wyzga N, Kreutzer DL, Justinich CJ, Gronowicz GA. Alterations in bone metabolism in children with inflammatory bowel disease an in vitro study. J Pediatr Gastroenterol Nutr 1997;24:289-95.

18. Issenman RM, Atkinson SA, Radoja C, Fraher L. Longitudinal assessment of growth, mineral metabolism and bone mass in pediatric Crohn's disease. J Pediatr Gastroenterol Nutr 1993;17:401-6.

19. Jeejeebhoy KN. The many faces of malnutrition in Crohn disease. Am J Clin Nutr 1988;67:819-20. 
Cabral VLR, Carvalho L de, Miszputen SJ. Importância da albumina sérica na avaliação nutricional e de atividade inflamatória em pacientes com doença de Crohn

20. Kishimoto T. The biology of interleukin-6. Blood 1989;74:1-10.

21. Kushner I. The phenomenon of the acute phase response. Ann N Y Acad Sci 1982;389:39-48

22. Lennard-Jones JE. Classification of inflammatory bowel disease. Scand J Gastroenterol 1989;24:2-6.

23. Mahida YR, Kurlac L, Gallaghera A, Hawkey CJ. High circulating concentrations of interleukin-6 in active Crohn's disease but not ulcerative colitis. Gut 1991;32:1531-4

24. Mendenhall CL, Tosch T, Weesner RE, Garcia-Pont P, Goldberg SJ, Kiernan T, Seeff LB, Sonell M, Tambuno C, Zetterman R, Chedid A, Chen T, Robin L. Veterans Administration Cooperative Study on Alcoholic Hepatitis II: prognostic significance of protein-calorie malnutrition. Am J Clin Nutr 1986;43:213-8.

25. Moum B, Ekbom A, Vatn MH, Savar AJ, Lygren I, Schulz T, Stray N, Fausa O. Inflammatory bowel disease: re-evaluation of the diagnosis in a prospective population - based study in south eastern Norway. Gut 1997;40:328-32.

26. Pullman WE, Elsbury S, Kobayashi M, Hapen AJ, Doe WF. Enhanced mucosal cytokine production in inflammatory bowel disease. Gastroenterology 1992;102:529-37.
27. Rothschild MA, Oratz M, Schreiber SS. Albumin synthesis (first of two parts). N Engl J Med 1972;286:748-57.

28. Sox HC, Liang MH. The erythrocyte sedimentation rate. Ann Intern Med 1986;104:515-23

29. Stokes MA. Crohn's disease and nutrition. Br J Surg 1992;79:391-4.

30. Strassmann G, Fong M, Kenney JS, Jacob CO. Evidence for the involvement of interleukin 6 in experimental cancer cachexia. J Clin Invest 1992;89:1681-4.

31. Suzuki Y, Saito H, Kasanuki J, Kishimoto T, Tamura Y, Yoshida S. Significant increase of interleukin 6 production in blood mononuclear leukocytes obtained from patients with active inflammatory bowel disease. Life Sci 1990;47:2193-7.

32. Wing SS, Goldberg AL. Glucocorticoids activate the ATP-Ubiquiten-dependent proteolytic system in skeletal muscle during fasting. Am J Physiol 1993;264:E668-76.

Recebido em 4/9/2000 Aprovado em 4/12/2000. 